\title{
Theoretical Analysis of Substituent Effects on Building Blocks of Conducting Polymers: 3,4'-Substituted Bithiophenes
}

\author{
Ulrike Salzner* and Tanyel Kızıltepe \\ Department of Chemistry, Bilkent University, 06533 Bilkent, Ankara, Turkey
}

Received J uly 17, 1998

\begin{abstract}
Substituents are widely used to modify the properties of conducting polymers. To study substituent effects on energy levels and energy gaps systematically, $\mathrm{CH}_{3^{-}}, \mathrm{OH}-, \mathrm{NH}_{2^{-}}, \mathrm{CN}-$, and CCH-substituted bithiophenes were examined with density functional theory and NBO analysis. Total charges and $\pi$-electron densities were analyzed separately to examine $\pi$ - and $\sigma$-effects. Second-order perturbation theory was used to quantify conjugation in terms of orbital interactions. NBO orbital energies were employed to investigate the effect of alternating donor-acceptor substitution. Substituents in 3and 4-positions shift HOMO and LUMO levels in parallel and hardly influence HOMO-LUMO gaps. For level shifting the $\pi$-donating and $\pi$-accepting abilities are most important; electronegativity mainly influences the $\sigma$-orbitals and is less crucial in determining energy gaps. Alternating donoracceptor substitution leads to HOMO and LUMO energies that are average between those of the parent systems and has little effect on energy gaps.
\end{abstract}

\section{Introduction}

Since the discovery that polyacetylene can be made conducting upon doping ${ }^{1}$ and since the synthesis of chemically more stable polymers such as polythiophene, 2,3 polypyrrole, ${ }^{4}$ and polyaniline, ${ }^{5-7}$ enormous progress has been made in modifying the properties of conducting polymers for specific applications.8,9 Various kinds of substituents are in use for improving solubility, decreasing band gaps, increasing polarizabilities, and optimizing luminescence efficiencies.

Alkyl substituents on the backbone of polythiophene are often employed to increase solubility. ${ }^{10}$ Little is known, however, about substituent effects on band gaps. Regioregular alkylthiophenes were shown to possess band gaps smaller than that of polythiophene. ${ }^{11}$ Substitution at the $\beta$-position can, for instance, increase conjugation and conductivity indirectly by decreasing the extent of $\alpha-\beta$-coupling during polymerization. ${ }^{8}$ Polar substituents increase hydrophilicity. Electropolymerization of $\mathbf{1}$ (Chart 1 ) resulted in a polymer that is watersoluble and fully electroactive in aqueous solution. ${ }^{9,12}$

* To whom correspondence should be addressed. Tel.: (312) 2902122. Fax: (312) 290-4579. E-mail: salzner@fen.bilkent.edu.tr.

(1) Chiang, C. K.; Park, Y. W.; Heeger, A.J .; Shirakawa, H.; Louis,

E. J.; MacDiarmid, A. G. Phys. Rev. Lett. 1977, 39, 1098.

(2) Diaz, A. F. Chem. Scr. 1981, 17, 142.

(3) Tourillon, G.; Garnier, F. J . Electroanal. Chem. Interfacial Electrochem. 1982, 135, 173.

(4) Diaz, A. F.; Kanazawa, K. K.; Gardini, G. P. J . Chem. Soc., Chem. Commun. 1979, 635

(5) Diaz, A. F.; Logan, J. A. J. Electroanal. Chem. Interfacial Electrochem. 1980, 111, 111 .

(6) Genies, E. M.; Tsintavis, C.; Syed, A. A. Mol. Cryst. Liq. Cryst. 1985, 121, 181.

(7) McDiarmid, A. G.; Chiang, J . C.; Halpern, M.; Huang, H. S.; Mu, S. L.; Somasiri, N. L. D.; Wu, W.; Yaninger, S. I. Mol. Cryst. Liq. Cryst. 1985, 121, 173 .

(8) Roncali, J Chem. Rev, 1992 92, 711.

(9) Roncali, J. Chem. Rev. 1997, 97, 173.

(10) Rumbles, G.; Samuel, I. D. W.; Magnani, L.; Murray, K. A.; DeMello, A. J .; Crystall, B.; Moratti, S. C.; Stone, B. M.; Holmes, A. B.; Friend, R. H. Synth. Met. 1996, 76, 47.

(11) Chen, T.-A.; Rieke, R. D. Synth. Met. 1993, 60, 175.

(12) Kozaki, M.; Tanaka, S.; Yamashita, Y. J . Chem. Soc., Chem. Commun. 1992, 1137.

\section{Chart 1}

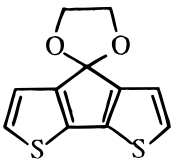

1<smiles>[R]C1=C([R])SC(=C2c3ccsc3-c3sccc32)S1</smiles>

4<smiles>c1ccc2cscc2c1</smiles>

2

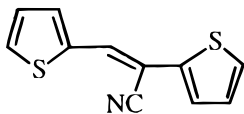

5<smiles>N#CC(C#N)=C1c2ccsc2-c2sccc21</smiles>

3<smiles>COc1ccsc1-c1sccc1C#N</smiles>

6
Grafting benzene onto thiophene rings yields quinoid polyisothianaphthene, the polymer of $\mathbf{2}$, having a $1 \mathrm{eV}$ smaller band gap than polythiophene. ${ }^{13}$ Another class of low-band-gap polymers is obtained by bridging bithiophene precursors with electron-withdrawing groups. ${ }^{14,15}$ In particular, dicyano groups such as those in $\mathbf{3}$ were shown to reduce the band gap from about $2 \mathrm{eV}$ in polythiophene to $0.8 \mathrm{eV} .{ }^{14-16}$ The band gap reduction is mainly due to lowering of the LUMO level, while the effect on the HOMO was found to be moderate. ${ }^{17}$ Dithia groups such as those shown in $\mathbf{4}$ have a similar effect, ${ }^{12}$ although the origin of this band gap reduction is not well-understood. ${ }^{9}$

(13) Wudl, F.; Kobayashi, M.; Heeger, A. J . J . Org. Chem. 1984, 49, 3382

(14) Ferraris, J . P.; Lambert, T. L. J . Chem. Soc., Chem. Commun. 1991, 1268.

(15) Lambert, T. L.; Ferraris, J . P. J . Chem. Soc., Chem. Commun. 1991, 752.

(16) Ferraris, J . P.; Lambert, T. L. J . Chem. Soc., Chem. Commun. 1991, 1268.

(17) Toussiant, J . M.; Brédas, J . L. Synth. Met. 1995, 69, 637. 
In contrast, introduction of a CN-substituted conjugated spacer group (cf. 5) decreases the band gap by raising the HOMO level. ${ }^{9,18}$ Alternating donor-acceptor units (6) are used to generate quantum well structures ${ }^{19-21}$ and to design polymers with low band gaps ${ }^{22,23}$ and large polarizabilities. ${ }^{23}$ Rigidification of polymer precursors by employing fused ring systems was expected to reduce band gaps by increasing order. ${ }^{9}$ However, band gap reduction was not generally observed.

Apart from desirable modification upon substitution, there are al so undesirable side effects. ${ }^{8}$ Bulky substituents induce deviations from planarity and decrease conjugation. Substituents such as the cyano group that lower the HOMO level of the monomer increase the oxidation potential required for electrochemical polymerization. In extreme cases the potentials required for electropolymerization are so drastic that monomers decompose rather than electropolymerize. If radicals formed during el ectropolymerization are too stable, they diffuse away from the el ectrode and short-chain oligomers rather than polymers are obtained. Conjugated precursors are especially hard to polymerize. Therefore, a substituent might change the structure and quality of the produced polymer to such an extent that analysis of intrinsic electronic effects of the substituent is difficult or impossible. Therefore, a detailed understanding of electronic effects is still lacking.

"Tailoring" of electronic structures requires knowledge of structure-property relationships. For this reason we have started theoretical investigations of substituent effects in different positions of heterocyclic polymer building blocks. Theoretical analysis of electronic structure-property relationships is not complicated by synthetic difficulties or by disorder effects encountered in experiment. Therefore, quite different systems can be examined on equal footing under identical conditions. In this paper we consider typical organic substituents: $-\mathrm{CH}_{3},-\mathrm{OH},-\mathrm{NH}_{2},-\mathrm{CN}$, and $-\mathrm{CCH}$ in the 3- and 4-positions of bithiophene (Chart 2). We have performed density functional calculations and electronic structure analyses which show the influence of substitution on band gaps, HOMO and LUMO levels, total charges, and charge densities in the $\pi$-systems. Delocalization of $\pi$-el ectrons al ong the conjugated backbone and between the backbone and the substituent was investigated. Donor-acceptor substitution was examined to analyze the nature of quantum well structures.

\section{Methods}

Compounds $\mathbf{7}$ through $\mathbf{1 3}$ were optimized using density functional theory (DFT). Becke's three-parameter hybrid functional ${ }^{24}$ was combined with Perdew and Wang's correlation functional. ${ }^{25}$ Stevens-Basch-Krauss pseudopotentials ${ }^{26}$ and

(18) Ho, H. A.; Brisset, H.; Elandaloussi, E. H.; Frére, P.; Roncali, J. Adv. Mater. 1996, 8, 990 .

(19) Kao, J.; Lilly, A. C., J r. J . Am. Chem. Soc. 1987, 109, 4149.

(20) Bakhshi, A. K.; Ladik, J .J .; Seel, M. Phys. Rev. 1987, B35, 704

(21) Suzuki, Y. Y.; Beljonne, D.; Brédas, J . L. J . Chem. Phys. 1996, $104,7270$.

(22) Bakhshi, A. K.; Yamaguchi, Y.; Ago, H.; Yamabe, T. Synth. Met. 1996, 79, 115.

(23) Demanze, F.; Yassar, A.; Garnier, F. Adv. Mater. 1995, 7, 907.

(24) Becke, A. D. Phys. Rev. A 1988, 38, 3098.

(25) Perdew, J. P. Phys. Rev. B 1986, 33, 8822. 6026.

\section{Chart 2}<smiles>c1csc(-c2cccs2)c1</smiles>

7<smiles>Cc1csc(-c2sccc2C)c1</smiles>

8<smiles>Nc1csc(-c2sccc2N)c1</smiles>

10

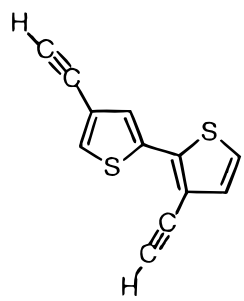

12<smiles>Oc1csc(-c2sccc2O)c1</smiles>

9

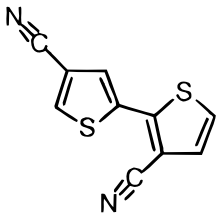

11

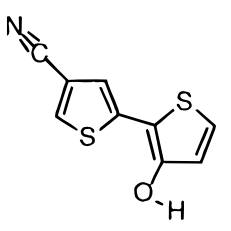

13 split valence plus pol arization basis sets were employed. ${ }^{27} \mathrm{All}$ molecules were optimized in a planar geometry. With the slightly pyramidal amino substituents no symmetry was imposed, but the inter-ring dihedral angle was fixed to maintain planarity of the backbone. This is justified in connection with conducting polymers, since it is known that thiophene oligomers are planar in the crystal, ${ }^{28}$ although the gas-phase structure is twisted by about $34^{\circ} .^{29}$

Use of DFT orbital energies for estimating ionization potentials, el ectron affinities, and energy gaps is a controversial subject ${ }^{30-39}$ and is beyond the scope of the present investigation. However, we have shown empirically in three recent publications that hybrid functionals greatly improve the

(27) Frisch, M J : Frisch, F. F Foresman, J. B. Gaussian 94 User's Reference; Gaussian, Inc.: Pittsburgh, PA, 1994-1995.

(28) Visser, G. J .; Heeres, G. J .; Wolters, J .; Vos, A. Acta Crystallogr., Sect. B 1968, 24, 467.

(29) Almenningen, A.; Gastiansen, O.; Suendas, P. Acta Chem. Scand. 1958, 12, 1671.

(30) Koopmans, T. A. Physica 1934, 1, 104.

(31) Baerends, E. J .; Gritsenko, O. V. J . Phys. Chem. A 1997, 101, 5383

(32) Fritsche, L. Physica B 1991, 172, 7.

(33) Godby, R. W.; Schlüter, M.; Sham, L. J . Phys. Rev. B 1988, 37, 10159.

(34) Levy, M. Phys. Rev. A 1995, 52, 50.

(35) Perdew, J. P.; Levy, M. Phys. Rev. Lett. 1983, 51, 1884

(36) Williams, A. R.: von Barth, U. Applications of Density Functional Theory to Atoms, Molecules, and Solids. In Theory of the Inhomogeneous Electron Gas; Lundqvist, S., March, N. H., Eds.; Plenum Press: London, 1983.

(37) Rösch, N.; Trickey, S. B. J . Chem. Phys. 1997, 106, 8940.

(38) Chan, G. E.-L.; Tozer, D. J .; Handy, N. C. J . Chem. Phys. 1997 107, 1536. 862.

(39) Galbraith, J . M.; Schaefer, H. F. I. J . Chem. Phys. 1996, 105 
Table 1. HOMO and LUMO Energies and HOMO-LUMO Gaps for 7-12 $\left(E_{g}\right)$ in eV

\begin{tabular}{lccc}
\hline & HOMO & LUMO & $\mathrm{E}_{\mathrm{g}}$ \\
\hline $\mathbf{7}$ & -6.58 & -1.78 & 4.81 \\
$\mathbf{8}$ & -6.41 & -1.58 & 4.84 \\
$\mathbf{9}$ & -6.21 & -1.63 & 4.58 \\
$\mathbf{1 0}$ & -5.96 & -1.40 & 4.56 \\
$\mathbf{1 1}$ & -7.66 & -3.02 & 4.64 \\
$\mathbf{1 2}$ & -6.77 & -2.24 & 4.52 \\
$\mathbf{1 3}$ & -6.88 & -2.09 & 4.79
\end{tabular}

performance of DFT with respect to band gaps. ${ }^{40-42}$ If the weight of the Hartree-F ock exchange is increased from 20 to $30 \%$, hybrid functionals yield HOMO-LUMO gaps in close agreement with $\lambda_{\max }$ values from UV spectroscopy. The modified functional is designated as B3P $86-30 \%$ and used in this publication throughout. IPs and EAs were shown to be about $1 \mathrm{eV}$ too low for oligomeric $\pi$-systems, but trends are reproduced correctly.

Substituent effects were analyzed in terms of localized orbitals with the NBO method, which is based on natural population analysis (NPA)..$^{43-45}$ The essential feature of NBO analysis is that the electron density is represented, as far as possible, by localized core orbitals, bonds, and lone pairs. These orbitals comprise L ewis structures corresponding precisely to the chemist's view of molecules built from atoms connected by localized two-electron bonds. However, for conjugated systems ideal Lewis structures are obviously not adequate. Deviations from idealized Lewis structures due to conjugation are shown in NBO analysis as orbital interactions between localized bonds and antibonds and between lone pairs and antibonds. The energetic contributions from these interactions can be quantified with the help of second-order perturbation theory (eq 1 ). $E^{2}$ is the second-order perturbational energy

$$
E^{2}=n \frac{\left\langle F_{i j}\right\rangle^{2}}{\epsilon_{i j}}
$$

stabilization. $F_{\mathrm{ij}}$ is the F ock matrix element between occupied orbital $\mathrm{i}$ and unoccupied orbital $\mathrm{j}$. $\mathrm{F}_{\mathrm{ij}}$ is proportional to the overlap between orbitals $\mathrm{i}$ and $\mathrm{j} . \epsilon_{\mathrm{ij}}$ is the energy difference between orbitals $i$ and $j$, and $n$ is the occupancy of orbital $i$. Thus, NBO analysis can be used to examine conjugation effects in $\pi$-conjugated systems quantitatively. Since perturbation theory is valid for small perturbations only, absolute stabilizations become less reliable as their values increase. Ther efore, we do not emphasize absolute numbers of stabilization energies but are concerned with trends. All calculations were performed with Gaussian $94 .{ }^{46}$

\section{Results}

Table 1 lists HOMO and LUMO energy levels and HOMO-LUMO gaps for unsubstituted bithiophene (7) and for compounds 8-13. In general, 3,4'-substitution of bithiophene has a moderate effect on HOMO-LUMO

(40) Salzner, U.; Lagowski, J . B.; Pickup, P. G.; Poirier, R. A. J . Comput. Chem. 1997, 18, 1943.

(41) Salzner, U.; Lagowski, J. B.; Pickup, P. G.; Poirier, R. A. J . Phys Chem. 1998, 102, 2572.

(42) Salzner, U.; Lagowski, J . B.; Poirier, R. A.; Pickup, P. G. Synth. Met. 1998, 96, 177-189.

(43) Reed, E. A.; Weinstock, R. B.; Weinhold, F. J . Chem. Phys. 1985 83, 735 .

(44) Reed, A. E.; Weinhold, F. J . Chem. Phys. 1985, 83, 1736.

(45) Reed, E. A.; Curtiss, L. A.; Weinhold, F. Chem. Rev. 1988, 88 899.

(46) Frisch, M. J .; Trucks, G. W.; Schlegel, H. B.; Gill, P. M. W.; J ohnson, B. G.; Robb, M. A.; Cheeseman, J. R.; Keith, T.; Petersson, G. A.; Montgomery, J. A.; Raghavachari, K.; Al-Laham, M. A. Zakrzewski, V. G.; Ortiz, J . V.; Foresman, J . B.; Peng, C. Y.; Ayala, P. Y.; Chen, W.; Wong, M. W.; Andres, J . L.; Replogle, E. S.; Gomperts, R.; Martin, R. L.; F Ox, D. J .; Binkley, J . S.; Defrees, D. J .; Baker, J .; Stewart, J . P.; Head-Gordon, M.; Gonzalez, C.; Pople, J . A. Gaussian 94, Revision B.3; Gaussian, Inc.: Pittsburgh, PA, 1995.
Table 2. NPA Charges of Heavy Atoms and of the Hydrogens of the Substituents

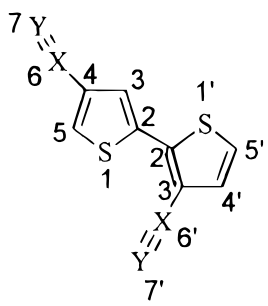

\begin{tabular}{|c|c|c|c|c|c|c|c|}
\hline & 7 & 8 & 9 & 10 & 11 & 12 & 13 \\
\hline C2 & -0.22 & -0.21 & -0.21 & -0.20 & -0.23 & -0.23 & -0.21 \\
\hline$C 2^{\prime}$ & -0.22 & -0.22 & 0.28 & -0.28 & -0.17 & -0.18 & -0.29 \\
\hline $\mathrm{C} 3$ & -0.22 & -0.22 & -0.30 & -0.27 & -0.16 & -0.18 & -0.19 \\
\hline $\mathrm{C}^{\prime}$ & -0.22 & -0.03 & +0.35 & +0.19 & -0.19 & -0.14 & +0.36 \\
\hline C4 & -0.22 & -0.04 & +0.33 & +0.19 & -0.19 & -0.15 & -0.19 \\
\hline$C 4^{\prime}$ & -0.22 & -0.22 & -0.29 & -0.26 & -0.18 & -0.19 & -0.29 \\
\hline C5 & -0.38 & -0.38 & -0.46 & -0.46 & -0.31 & -0.33 & -0.33 \\
\hline $\mathrm{C}^{\prime}$ & -0.38 & -0.37 & -0.36 & -0.36 & -0.36 & -0.37 & -0.35 \\
\hline $\mathrm{S} 1$ & +0.40 & +0.39 & +0.44 & +0.37 & +0.49 & +0.46 & +0.47 \\
\hline $\mathrm{S1}^{\prime}$ & +0.40 & +0.39 & +0.40 & +0.39 & +0.45 & +0.42 & +0.42 \\
\hline$\sum_{\text {backbone }}$ & -1.2 & -0.67 & -0.38 & -0.69 & -0.85 & -0.89 & -0.60 \\
\hline $\bar{x} 6$ & & -0.58 & -0.73 & -0.84 & +0.33 & -0.05 & +0.33 \\
\hline$X 6^{\prime}$ & & -0.58 & -0.73 & -0.84 & +0.31 & -0.07 & -0.73 \\
\hline Y7 & & & & & -0.35 & -0.17 & -0.37 \\
\hline Y $7^{\prime}$ & & & & & -0.34 & -0.15 & \\
\hline $\mathrm{H}$ & & $\begin{array}{r}0.20 / \\
0.21\end{array}$ & 0.50 & +0.38 & & +0.21 & +0.50 \\
\hline $\mathrm{H}$ & & $\begin{array}{r}0.20 / \\
0.21\end{array}$ & +0.49 & +0.38 & & +0.21 & \\
\hline ubs & & +0.04 & -0.23 & -0.12 & -0.02 & -0.01 & $-0.04 /$ \\
\hline
\end{tabular}

gaps. A slight energy gap reduction compared to bithiophene is obtained with $\mathrm{OH}, \mathrm{NH}_{2}, \mathrm{CN}$, and $\mathrm{CCH}$ groups. The HOMO-LUMO gaps of the symmetrically substituted molecules (9-12) are al most identical, differing by only $0.12 \mathrm{eV}$. With methyl and mixed CN/OH substitution the HOMO-LUMO energy separation remains essentially unchanged compared to bithiophene.

In contrast to HOMO-LUMO gaps, individual energy levels shift strongly. The HOMO of $\mathbf{1 1}$, for instance, which is the lowest of the molecules investigated, lies 1.7 $\mathrm{eV}$ lower than that of 10. Methyl, hydroxy, and amino groups increase the HOMO and LUMO energies and therefore result in lower IPs and EAs. ${ }^{30}$ Cyano and acetylene groups shift both levels down. For mixed CN/ $\mathrm{OH}$ substitution the two opposite effects partially compensate but the total effect is closer to that of $\mathrm{OH}$ rather than to that of $\mathrm{CN}$ substitution. In all molecules the change upon substitution in HOMO energy is similar to the change in LUMO energy, resulting in almost no change to the energy gap, as described above.

Table 2 lists charges obtained with natural population analysis (NPA). Sulfur atoms are positively charged, while carbon atoms of the backbone are negatively charged. The negative charges on the carbons are mainly due to the polarity of the $\mathrm{C}-\mathrm{H}$ bonds. Replacing $\mathrm{H}$ by $\mathrm{CH}_{3}$ results in almost neutral carbons ( $\mathrm{C}^{\prime}$ and $\mathrm{C} 4$ in 8 ). Carbon atoms that carry $\mathrm{OH}$ or $\mathrm{NH}_{2}$ substituents ( $\mathrm{C}^{\prime}$ and $\mathrm{C} 4$ in 9 and 10) have positive charges. $\mathrm{CN}$ - and $\mathrm{CCH}$ substituted carbons are negatively charged. Oxygen and nitrogen atoms carry large negative charges. Comparison with the charges of the hydroxy and amino hydrogens reveals that the extra electrons on $\mathrm{O}$ and $\mathrm{N}$ are mainly withdrawn from the hydrogens rather than from the bithiophene system. On the bottom of Table 2 the total charges for the $\mathrm{CH}_{3}, \mathrm{OH}, \mathrm{NH}_{2}, \mathrm{CN}$, and $\mathrm{CCH}$ substituents are shown. These numbers reveal that the $\mathrm{OH}$ group only withdraws 0.23 electron from the bithiophene backbone 
Table 3. Electron Density in the $\pi$-Systems of 7-12

\begin{tabular}{llllllll}
\hline & \multicolumn{1}{c}{$\mathbf{7}$} & \multicolumn{1}{c}{$\mathbf{8}$} & \multicolumn{1}{c}{$\mathbf{9}$} & \multicolumn{1}{c}{$\mathbf{1 0}$} & \multicolumn{1}{c}{$\mathbf{1 1}$} & \multicolumn{1}{c}{$\mathbf{1 2}$} & $\mathbf{1 3}$ \\
\hline $\mathrm{C}=\mathrm{C}$ & 8.66 & 8.65 & 8.85 & 8.82 & 8.70 & 8.63 & 8.74 \\
charge & -0.66 & -0.65 & -0.85 & -0.82 & -0.70 & -0.63 & -0.74 \\
$\mathrm{~S} 1 / \mathrm{S1}^{\prime}$ & $1.64 / 1.64$ & $1.65 / 1.65$ & $1.66 / 1.64$ & $1.67 / 1.68$ & $1.61 / 1.59$ & $1.61 / 1.63$ & $1.60 / 1.65$ \\
$\sum_{\pi}$ & 11.94 & 11.95 & 12.15 & 12.17 & 11.90 & 11.87 & 11.99 \\
charge & +0.06 & +0.05 & -0.15 & -0.17 & +0.10 & +0.13 & +0.01
\end{tabular}

Table 4. Strengths of Interactions between Occupied and Formally Unoccupied NBOs in $\mathrm{kcal} / \mathrm{mol}$

\begin{tabular}{|c|c|c|c|c|c|c|}
\hline & 7 & 8 & 9 & 10 & 11 & 12 \\
\hline$\sum_{\pi-\pi^{*}}$ & 105 & 104 & 105 & 103 & 104 & 104 \\
\hline$\sum_{\mid p S-\pi^{*}}$ & 101 & 96 & 102 & 94 & 105 & 101 \\
\hline$\sum_{\mathrm{X}-\pi^{*}}$ & & 18 & 62 & 58 & 19 & 27 \\
\hline$\sum_{\pi-X^{*}}$ & & 10 & & & 35 & 33 \\
\hline$\sum_{\mathrm{X}-\pi^{*}-\pi-\mathrm{X}^{*}}$ & & 8 & 62 & 58 & -16 & -6 \\
\hline
\end{tabular}

and the amino group only withdraws 0.12 electron. The total electron-withdrawing effect of the cyano and acetylene groups is negligible. The only positively charged substituent is the methyl group, but the charge is very small (0.04 e). In the mixed substituted compound $13 \mathrm{OH}$ and $\mathrm{CN}$ behave very similar to the symmetrically substituted compounds $\mathbf{9}$ and $\mathbf{1 1}$.

In Table 3 the charges of the $\pi$-system are evaluated. The charges in the $\pi$-system reveal effects that are hidden in the total charges. The total charges of the bithiophene framework are all negative, mainly due to the polarity of the $\mathrm{C}-\mathrm{H}$ bonds. However, the $\pi$-system can be positively or negatively charged depending on the substituent. The total charges in the last line of Table 4 show that the $\pi$-backbone has a surplus of el ectrons with $\mathrm{OH}$ and $\mathrm{NH}_{2}$ substituents but is positively charged with $\mathrm{CN}$ and $\mathrm{CCH}$ substituents. Mixing $\mathrm{OH}$ and $\mathrm{CN}$ yields a neutral $\pi$-backbone. Breaking down the $\pi$-charge into contributions from the carbon framework and the sulfur $\pi$-orbitals shows that sulfur acts as a strong $\pi$-electron donor. The carbon framework has negative charges between -0.85 and -0.63 . Where the backbone is more negative due to substitution, the sulfur $\pi$-lone pairs have larger occupancies, indicating that the sulfur lone pairs tend to compensate the electronic effects of the substituents.

To investigate the origin of the different $\pi$-electron densities, we evaluated the strengths and direction of electronic effects within the $\pi$-backbone, between backbone and sulfur lone pairs, and between the backbone and substituents. The stabilization energies resulting from the corresponding donor-acceptor interactions (eq 1) are listed in Table 4. The first line of Table 4 shows that the sum of energetic stabilizations within the bithiophene backbone hardly changes upon substitution. In contrast, the interactions of the sulfur lone pairs with the backbone differ by up to $11 \mathrm{kcal} / \mathrm{mol}$. With bithiophene as a reference, we can infer that methyl and amino substitution decrease $\pi$-donation from sulfur to the backbone, hydroxy and acetylene groups leave the interaction unchanged, and cyano substitution increases donation from sulfur lone pairs. Equation 1 shows that stabilization energies increase if the energy difference between the orbitals decreases and if the overlap increases. The changes observed here are entirely due to overlap differences, while energy differences are constant.

The strongest differences are observed for interactions between substituents and the backbone. Methyl groups act as $\pi$-donors and $\pi$-acceptors. The $\pi$-donating ability is slightly stronger than the $\pi$-accepting capacity. There-
Table 5. Energies of Localized $\pi$-NBOs in eV

\begin{tabular}{lccccccc}
\hline & $\mathbf{7}$ & $\mathbf{8}$ & $\mathbf{9}$ & $\mathbf{1 0}$ & $\mathbf{1 1}$ & $\mathbf{1 2}$ & $\mathbf{1 3}$ \\
\hline C2-C3 $^{\prime}$ & -8.27 & -8.11 & -8.19 & -8.00 & -9.28 & -8.52 & -8.69 \\
C2 $^{\prime}-$ C3 $^{\prime}$ & -8.27 & -8.05 & -8.49 & -8.09 & -9.37 & -8.53 & -8.86 \\
C4-C5 & -8.42 & -8.17 & -8.24 & -8.13 & -9.39 & -8.54 & -8.90 \\
C4-C5' $^{\prime}$ & -8.42 & -8.34 & -8.73 & -8.42 & -9.40 & -8.76 & -9.02
\end{tabular}

fore, a net $\pi$-donating effect results. This kind of interaction of methyl groups with conjugated $\pi$-systems was first discovered by Mulliken and was termed hyperconjugation. ${ }^{47-50} \mathrm{H}$ ydroxy groups, due to their $\pi$-lone pairs, are much stronger donors than methyl groups. Since there are no suitable acceptor or bitals, hydroxy groups cannot accept electrons from the $\pi$-system. Therefore, hydroxy groups are strong $\pi$-donors. The same holds for amino substituents. Cyano and acetylene groups interact with the $\pi$-backbone through their $\pi$ - and $\pi^{*}$-orbitals. For the acetylene group $\pi$-donating and $\pi$-accepting abilities are similar, with a slight preference for electron acceptance. With cyano groups the electron-accepting capability dominates, making the cyano group a $\pi$-acceptor.

Cyano groups withdraw electrons from the $\pi$-system and thereby lower HOMO and LUMO energy levels. Hydroxy groups donate electrons and raise HOMO and LUMO energy levels. To see whether there are alternating high and low localized $\pi$-energy levels in donoracceptor-substituted bithi ophene, we compiled $\pi$-energy levels of NBOs. These values are given in Table 5. In unsubstituted bithiophene the energies of the inner $\pi$-bonds are higher than those of the outer bonds. This reflects the end effects and the weaker involvement of the outer bonds in $\pi$-conjugation. In 8-12 there is bond alternation due to the unsymmetrical substitution pattern and end effects. In particular, the unsubstituted outer $\pi$-bond lies lower in energy than the other $\pi$-bonds which are involved in conjugation. Donor-acceptor substitution does not enhance this bond alternation. Moreover, all $\pi$-energies in $\mathbf{1 3}$ are roughly the average of the corresponding levels in $\mathbf{9}$ and $\mathbf{1 1}$.

\section{Discussion}

Trends in HOMO-LUMO gaps and $\pi$-conjugation calculated for dimers 7-13 should be valid for the corresponding polymers, since polymer properties devel op gradually from monomer properties as the chain lengths increase. Final band gaps depend on how small HOMOLUMO gaps of the building blocks are and on how fast the gaps decrease as chain lengths increase. We have examined various heterocyclic oligomers and found that the slopes of HOMO-LUMO gaps plotted versus inverse chain lengths are very similar. 41,42 In no case did we observe an inversion, that is, that a monomer with a larger HOMO-LUMO gap yielded a polymer with a

(47) Mulliken, R. S. J . Chem. Phys. 1933, 1, 491.

(48) Mulliken, R. S. J . Chem. Phys. 1935, 3, 520.

(49) Mulliken, R. S. J . Chem. Phys. 1939, 7, 339.

(50) Mulliken, R. S.; Rieke, C. A.; Brown, W. G. J . Am. Chem. Soc 1941, 63, 41. 
smaller band gap compared to a monomer with a smaller HOMO-LUMO gap. Therefore, trends in HOMOLUMO gaps observed for dimers are expected to be representative for trends in band gaps of the corresponding polymers.

In a classical approximation, the conductivity of conducting polymers is related to the number of charge carriers $\mathrm{n}$ and the mobility of these charge carriers, which depends on the time between collisions $\tau$ (eq 2). e is the

$$
\sigma=\frac{\mathrm{ne}^{2} \tau}{\mathrm{m}}
$$

elementary charge, and $m$ is the mass of the charge carriers. The charge carrier concentration increases as the energy separation between the valence and conduction bands decreases. The mobility of the charge carriers is influenced by the bandwidth, which reflects the overlap and therefore the extent of $\pi$-conjugation.

The fact that the HOMO-LUMO energy differences are only slightly affected by substitution in the 3- and 4-positions of bithiophenes 8-13 indicates that substituents in these positions have little effect on polymer band gaps. NBO analysis reveals (compare Table 4) that $\pi$-conjugation al ong the carbon backbone is also hardly affected by 3,4'-substitution. Therefore, a dramatic change in conductivity upon 3,4'-substitution should not be expected. This is in agreement with experiment, which did not reveal clear trends in conductivity depending on substitution. ${ }^{8}$

The strong effect of substitution on HOMO and LUMO levels shows that polymers of 7-13 will have very different IPs and EAs. Therefore, substituents in 3- and 4-positions can be used to design n-dopable polymers. However, strong energy lowering effects, as in the case of cyano groups, have the disadvantage that the monomers cannot be electropolymerized due to their large $\mathrm{IPs}^{23}$

The lowering of energy levels with the cyano group is usually attributed to its large group electronegativity ( 3.5 on the Pauling scale). According to the Pauling definition, electronegativity is "the power of an atom or group to attract shared el ectrons to itself." ${ }^{\prime 1}$ I t is therefore surprising to find that the cyano group has a charge of only -0.02 e in $\mathbf{1 1}$ and that the $\mathrm{C}-\mathrm{CN}$ bond is almost perfectly covalent according to NBO analysis. Considering that we are dealing with a carbon-carbon bond, the absence of polarity is understandable. In contrast, hydroxy and amino groups withdraw electrons from the bithiophene backbone and are negatively charged, as expected for electronegative groups. However, hydroxy and amino groups raise rather than lower the $\pi$-energy levels, which is in contrast with photoelectron spectroscopy, which shows that binding energies increase when el ectrons are withdrawn. These apparent contradictions can be resolved by breaking down charges into $\pi$ - and $\sigma$-contributions. Table 3 shows that the trends in the $\pi$-charges are in perfect agreement with relative $\pi$-energy levels. Hydroxy and amino groups increase the $\pi$-density and raise HOMO and LUMO levels; cyano and acetylene groups withdraw $\pi$-electrons and lower $\pi$-energy levels. The methyl group has little effect on the $\pi$-electron density. It slightly (0.01 e) decreases the positive charge

(51) Pauling, L. The Nature of the Chemical Bond, 2nd ed.; Oxford Univ. Press: London, 1958; p 58.
Table 6. Strengths of $\pi-\pi^{*}$-Interactions and Energy Differences and Fock Matrix Elements between Donor and Acceptor Orbitals

\begin{tabular}{lccc}
\hline & $\mathrm{E}^{2}$ & $\mathrm{e}_{\mathrm{j}}$ & $\left\langle\mathrm{F}_{\mathrm{ij}}\right\rangle$ \\
\hline & $\mathrm{CN}$ & & \\
$\pi_{\mathrm{C} 2^{\prime} \mathrm{C} 3^{\prime} \rightarrow \pi^{*} \mathrm{C} 6^{\prime} \mathrm{N} 7^{\prime}}$ & 17.75 & 0.43 & 0.081 \\
$\pi_{\mathrm{C} 6^{\prime} \mathrm{N} 7^{\prime} \rightarrow \pi^{*} \mathrm{C} 2^{\prime} \mathrm{C}^{\prime}}$ & 9.74 & 0.38 & 0.060 \\
& $\mathrm{CCH}$ & & \\
$\pi_{\mathrm{C} 2^{\prime} \mathrm{C} 3^{\rightarrow} \rightarrow \pi^{*}{ }^{*} 6^{\prime} \mathrm{N} 7^{\prime}}$ & 16.40 & 0.42 & 0.077 \\
$\pi_{\mathrm{C} 6^{\prime} \mathrm{N} 7^{\prime} \rightarrow \pi^{*} \mathrm{C} 2^{\prime} \mathrm{C} 3^{\prime}}$ & 13.61 & 0.34 & 0.067
\end{tabular}

Scheme 1
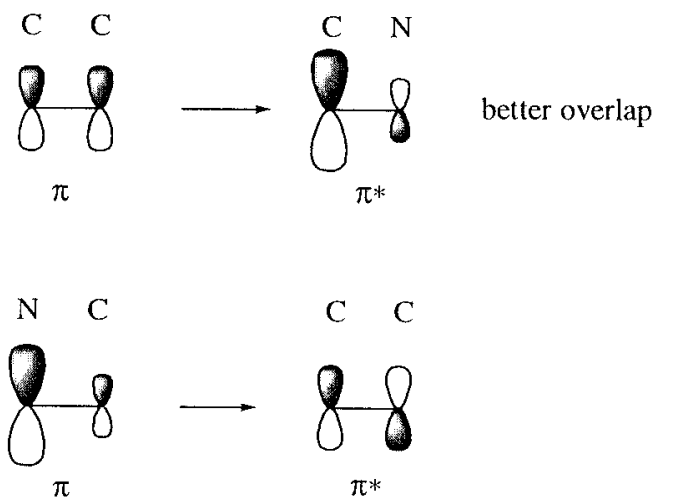

of the $\pi$-backbone compared to bithiophene and slightly raises the energy levels compared to bithiophene.

The $\pi$-donating and $\pi$-withdrawing effects can be understood in terms of relative donor and acceptor strengths, as obtained with eq 1 (Table 4). For the methyl group $\pi$-donation and $\pi$-acceptance are competitive and a small overall $\pi$-donating effect results. Hydroxy and amino groups interact with the $\pi$-system through their Ione pairs and do not have suitable acceptor orbitals. Therefore, they can only donate, not accept, $\pi$-electrons. Strong overall $\pi$-donation effects result. For all three substituents $\pi$-charges and total charges have opposite signs. This means these substituents are $\pi$-donors but $\sigma$-acceptors. The analyses of the energy levels above show that for conducting polymers $\pi$-effects are more important, since band gaps and bandwidths are properties of the $\pi$-system. Thus, in connection with conducting polymers, methyl, hydroxy, and amino groups can be considered as electron donors. Electronegativity is of secondary importance. With acetylene and cyano groups, the situation is reversed. Both groups donate $\sigma$-electrons and withdraw $\pi$-electrons with little effect on the total charges.

To understand the $\pi$-withdrawing effect of the cyano group better, we compare cyano and acetylene groups. The acetylene group is also covalently bound and also has a triple bond next to the bithiophene backbone. In Table 6 , the two types of $\pi-\pi^{*}$-interactions are compared for $\mathbf{1 1}$ and $\mathbf{1 2}$. NBO analysis shows that for both groups the $\pi$-accepting ability is stronger than the $\pi$-donating ability, since the overlap is larger between $\pi_{\mathrm{CC}}$ and $\pi^{*}{ }_{\mathrm{CN}(\mathrm{CCH})}$ than between $\pi_{\mathrm{CN}(\mathrm{CCH})}$ and $\pi^{*} \mathrm{CC}$. The energy differences between donor and acceptor would favor the opposite situation. This overlap difference is stronger for $\mathrm{CN}$ than for $\mathrm{CCH}$. Thus, it is the polarity of the $\mathrm{CN}$ triple bond, as sketched in Scheme 1 that makes the cyano group a $\pi$-accepting substituent.

The origin of the electron-withdrawing and $\pi$-energylowering effect of the cyano group is important for the understanding of similar effects involving dithia groups 
in polymers of $\mathbf{4} .^{12}$ The similarity was not understood, since the effect of the cyano group was attributed to its "Iarge group el ectronegativity" and the absence of such an electronegativity effect with sulfur. ${ }^{9}$ The above discussion, however, shows that the effect of the cyano group is quite different from the traditional view of el ectronegativity. It is in fact due to the presence of a polar $\pi$-bond. That acetylene groups have a similar, albeit weaker, effect, allows us to rationalize the similarity between dicyano and dithia groups.

The positive $\pi$-charge of the backbone with the cyano group induces a lower lone pair occupation on sulfur. With $\pi$-electron donors such as hydroxy and amino groups, the sulfur lone pairs have higher occupancies than in bithiophene and in $\mathbf{1 1}$ and $\mathbf{1 2}$. Thus, the sulfur Ione pairs partially compensate for the electron-withdrawing and -donating effects of substituents. This indicates that there is a possibility that polymers with donors less effective than sulfur, e.g. polycycl opentadiene, polysilole, or polyphosphole, will show greater response to substitution.

Since hydroxy groups are strong $\pi$-donors and raise $\pi$-energy levels and cyano groups are strong $\pi$-acceptors and lower $\pi$-energy levels, it was suggested ${ }^{22}$ that alternating donor-acceptor units could be employed to lower band gaps and to create quantum-well structures. $20,22,23,52,53$ Comparison of localized $\pi$-energy levels in $\mathbf{1 3}$ with those in $\mathbf{9}$ and $\mathbf{1 1}$ shows that substituent effects average out due to the delocalized nature of the $\pi$-system. Alternating donor-acceptor substitution does therefore not produce quantum-well structures in the ground state of this type of conjugated organic polymers. Additivity of electronic effects also has been observed experimentally for CN/ OMe-substituted bithiophene, ${ }^{23}$ which has an oxidation potential that differs only by $0.03 \mathrm{~V}$ from that of bithiophene. Nonetheless, the obtained polymer had interesting electrochemical properties upon oxidation. Our result also is in agreement with findings that donoracceptor substitution shows stronger effects if larger blocks of donors and acceptors are used. ${ }^{54}$ If the blocks are large enough, it is reasonable to expect that properties of the pure polymers are reached in the middle of the blocks and that alternating high and low energy levels can be realized. The absence of donor-acceptor

(52) Yamamoto, T.; Zhou, Z.; Kanbara, T.; Shimura, M.; Kizu, K.; Maruyama, T.; Nakamura, Y.; Fukuda, T.; Lee, B.-L.; Ooba, N.; Tomaru, S.; Kurihara, T.; Kaino, T.; Kubota, K.; Sasaki, S. J . Am. Chem. Soc. 1996, 118, 10389.

(53) Mullekom, H. A. M. v.; Vekemans, J . A. J . M.; Meijer, E. W. Chem. Commun. 1996, 2163.

(54) Alemán, C.; Domingo, V. M.; Fajarí, L.; J uliá, L.; Karpfen, A. J. Org. Chem. 1998, 63, 1041. effects in neighboring donor-acceptor oligomers, however, applies strictly to ground states and does not rule out effects on excitation spectra, electrochemical properties, and polarizabilities. For successful designing of lowband-gap polymers with donor-acceptor moieties, however, symmetry considerations seem to be crucial. If substituents can be placed in a position where the HOMO and LUMO are affected differently, than one of the levels can be shifted with respect to the other and band gaps decrease. This was demonstrated for bridged thiophenes such as $\mathbf{3} \cdot 14,15,17,22$

\section{Conclusions}

Substitution of conjugated $\pi$-systems in the 3- and 4-positions is predicted to have a small effect on energy gaps and on $\pi$-conjugation. Therefore, we expect little effect on conductivity. Since HOMO and LUMO levels are shifted strongly by 3- and 4-substitution, $\pi$-electronwithdrawing substituents can be used to design ndopable polymers. Alternating donor-acceptor substitution leads to averaging of energy levels and does not produce quantum-well structures in the ground state of our models.

For designing of low-band-gap materials with donoracceptor moieties, it is crucial to consider the symmetries of the HOMO and LUMO and to place substituents in such a way that HOMO and LUMO levels are influenced individually. One such strategy is to substitute in the bridging position, as in $\mathbf{3}$ and $\mathbf{4}$. Since the HOMO has a node in that position, the LUMO is affected more strongly and band gaps can be engineered. Studies of bridged dimers are underway.

The effect of substituents on energy gaps, IPs, and EAs is dominated by their relative $\pi$-donating and $\pi$-accepting power. Electronegativities are of secondary importance. The "electron withdrawing effect" and the "large electronegativity" of the cyano group are better analyzed in terms of hyperconjugative effects. Due to the polarity of the $\mathrm{CN}$ triple bond, the overlap is larger between $\pi_{\mathrm{Cc}}$ and $\pi^{*}{ }_{\mathrm{CN}}$ than between $\pi_{\mathrm{CN}}$ and $\pi^{*} \mathrm{CN}$. This leads to domination of the former orbital interaction over the latter, making $\mathrm{CN}$ a $\pi$-acceptor. However, the $\mathrm{C}-\mathrm{CN}$ bond is perfectly covalent and $\mathrm{CN}$ does not "attract shared electrons to itself." The carbon bound to the dicyanomethylene group is, in fact, negatively charged due to $\sigma$-effects. The interpretation of the effect of the cyano group in terms of hyperconjugation, on the other hand, makes plausible why similar effects are observed with the $\mathrm{C}=\mathrm{C}(\mathrm{CN})_{2}$ and $\mathrm{C}=\mathrm{C}(\mathrm{SR})_{2}$ groups.

J 09813944 